\title{
The incidences and mortalities of major cancers in China, 2009
}

\author{
Wanqing Chen"1, Rongshou Zheng ${ }^{1}$, Siwei Zhang ${ }^{1}$, Ping Zhao', Guanglin Li², \\ Lingyou $\mathrm{Wu}^{2}$ and Jie $\mathrm{He}^{1}$
}

Abstract

In 2012, the National Central Cancer Registry (NCCR) of China collected cancer registration information for the year 2009 from local cancer registries and analyzed it to describe the incidences and mortalities of cancers in China. Based on the data quality criteria from NCCR, data from 104 registries covering $85,470,522$ people $(57,489,009$ in urban areas and $27,981,513$ in rural areas) were checked and evaluated. The data from 72 registries were qualified and accepted for the cancer registry annual report in 2012. The total cancer incident cases and cancer deaths were 244,366 and 154,310 , respectively. The morphologically verified cases accounted for $67.23 \%$, and $3.14 \%$ of the incident cases only had information from death certifications. The crude incidence in the Chinese cancer registration areas was 285.91/ 100,000 (317.97/100,000 in males and 253.09/100,000 in females). The age-standardized rates for incidences based on the Chinese standard population (ASRIC) and the world standard population (ASRIW) were 146.87/100,000 and 191.72/100,000, respectively, with a cumulative incidence of $22.08 \%$. The cancer mortality in the Chinese cancer registration areas was $180.54 / 100,000 \quad(224.20 / 100,000$ in males and 135.85/100,000 in females). The age-standardized rates for mortalities based on the Chinese standard population (ASRMC) and the world standard population (ASRMW) were 85.06/100,000 and $115.65 / 100,000$, respectively, and the cumulative mortality was $12.94 \%$. Lung cancer, gastric cancer, colorectal cancer, liver cancer, esophageal cancer, pancreatic cancer, encephaloma, lymphoma, female breast cancer, and cervical cancer were the most common cancers, accounting for $75 \%$ of all cancer cases. Lung cancer, gastric cancer, liver cancer, esophageal cancer, colorectal cancer, pancreatic cancer, breast cancer, encephaloma, leukemia, and lymphoma accounted for $80 \%$ of all cancer deaths. The cancer registration's population coverage has been increasing, and its data quality is improving. As the basis of the cancer control program, the cancer registry plays an important role in directing anticancer strategies in the medium and long term. Because cancer burdens are different in urban and rural areas in China, prevention and control efforts should be based on practical situations.

Key words Cancer registry, incidence, mortality, epidemiology, China

Population-based cancer registries collect the data of cancer incident cases and deaths from the covered

Authors' Affiliations: 'National Office for Cancer Prevention and Control, National Cancer Center, Beijing 100021, P. R. China; ${ }^{2}$ Bureau of Disease Prevention and Control, Ministry of Health, Beijing 100044, P. R. China

Corresponding Author: Wanqing Chen, National Office for Cancer Prevention and Control, National Cancer Center, Beijing 100021, P. R. China. Email: chenwq@ cicams.ac.cn.

*The full text article of this paper is first published in the Chinese Journal of Cancer Research (2013, 25:10-21); the main content is published as a Brief Report in the Chinese Journal of Cancer with permission. doi: $10.5732 /$ cjc. 013.10018 populations to describe and monitor cancer epidemics in certain areas. The cancer registration data are used for cancer control planning, for the implementation and evaluation of cancer prevention and control efforts, and for scientific research ${ }^{[1]}$. Since 2006, when the Disease Prevention and Control Bureau, Ministry of Health of China started to publish cancer statistics annually, the National Central Cancer Registry (NCCR) of China has been responsible for collecting data from all local registries, calculating the statistical items accurately, analyzing the data of cancer incident cases and deaths from registration areas, and releasing the updated data in the Cancer Registry Annual Report ${ }^{[2]}$. The cancer 
statistical data have been broadly used in scientific studies, clinical trials, and decision-making for cancer prevention and control strategies.

\section{Materials and Methods}

\section{Data source}

One hundred four cancer registries from 26 provinces, including 46 urban registries and 58 rural registries, submitted cancer registration data in 2009 . The coverage population of all 104 registries was $109,476,347(55,654,485$ males and $53,821,862$ females), accounting for $8.20 \%$ of the entire national population by the end of 2009. The total cancer incident cases were $284,470(160,958$ males and 123,512 females), and the total number of deaths was $174,879(110,311$ males and 64,568 females).

\section{Quality control}

The proportion of morphologic verification (MV\%), the percentage of cancer cases identified by death certification only (DCO\%), the mortality-to-incidence ratio $(\mathrm{M} / \mathrm{I})$, the percentage of uncertified cancer (UB\%), and the percentage of cancer with undefined or unknown primary site (secondary) (O\&U\%) were used to evaluate the completeness, validity, and reliability of the cancer registration data. According to NCCR's acceptability criteria, the following standards should be reached: $\mathrm{MV} \%$ higher than $66 \%, \mathrm{DCO} \%$ less than $15 \%$, and $\mathrm{M} / \mathrm{l}$ ratio between 0.6 and 0.8 .

An advantage of cancer registration data is the timely reporting of cancer. However, for the completeness, validity, and reliability of cancer statistics ${ }^{[3]}$, a time gap between data updating and analysis might exist. NCCR ruled that every registry should upload the cancer registry data for 2009 before July 1, 2012, which was 30 months after the reported patients' cancer diagnoses were made.

\section{Statistical analysis}

The quality of the data was assessed based on the "Guideline for Chinese Cancer Registration" and referred to the criteria for "Cancer Incidence in Five Continents Volume IX" ${ }^{[4]}$ by the International Agency for Cancer Registry (IACR) and the International Agency for Research on Cancer (IARC), respectively. When the cancer registration data met the quality criteria for completeness, comparability, and validity, they were accepted as qualified data for analysis.

Crude incidence and mortality were calculated and age-standardized to the 1982 Chinese population and Segi's world population. The proportion and cumulative rate were also calculated. Database software, including MS-FoxPro, MS-Excel, SAS, and IARC issued by IARC/IACR ${ }^{[5]}$, were used for data checking, evaluation, and analysis.

\section{Results}

\section{Data pooling and quality evaluation}

The data from 72 registries, including 31 from urban areas and 41 from rural areas, met the criteria for data quality and were pooled to create a national database in 2009 . The population coverage of the valid database was $85,470,522(43,231,554$ males and $42,238,968$ females), including $57,489,009$ in urban areas $(67.26 \%)$ and $27,981,513$ in rural areas $(32.74 \%)$. The total cancer incident cases and deaths were 244,366 and 137,462, respectively (Table 1 ).

The overall $\mathrm{MV} \%$, DCO $\%$, and $\mathrm{M} / \mathrm{I}$ ratio were $67.23 \%$, $3.14 \%$, and 0.63 , respectively. They were $68.96 \%, 3.03 \%$, and 0.60 in urban registries, compared with $62.91 \%, 3.43 \%$, and 0.71 in rural registries.

\section{Incidence and mortality of all cancers}

\section{Incidence}

The crude incidence of all cancers in the registration areas was 285.91/100,000 (317.97/100,000 in males and $253.09 / 100,000$ in females). The ASIRC was $146.87 / 100,000$, and the ASIRW was 191.72/ 100,000 . Among the patients aged $0-74$ years, the cumulative incidence was $22.08 \%$. The crude and age-standardized cancer incidences in urban areas were higher than those in rural areas. Although the crude incidence in males was much higher in urban areas than in rural areas, the age-standardized incidences were similar (Table 2).

\section{Mortality}

The crude mortality in the cancer registration areas was $180.54 / 100,000 \quad(224.20 / 100,000$ in males and $135.85 / 100,000$ in females). The ASMRC was 85.06/ 100,000 , and the ASMRW was $115.65 / 100,000$. The cumulative mortality (0-74 years) was $12.94 \%$. Urban areas had a higher cancer mortality $(181.86 / 100,000)$ than rural areas did $(177.83 / 100,000)$, and both rural and urban areas had similar mortality for males. After age standardization, the mortality in rural areas was higher than that in urban areas for both males and females (Table 3).

\section{Incidence and mortality of major cancers}

Cancer incidences of the 10 most common cancers Lung cancer was the most common cancer in the 


\begin{tabular}{|c|c|c|c|c|c|c|c|c|c|c|}
\hline \multirow{2}{*}{$\begin{array}{l}\text { Cancer } \\
\text { Registry }\end{array}$} & \multirow{2}{*}{$\begin{array}{c}\text { Category } \\
\text { 1: urban; } 2 \text { : rural }\end{array}$} & \multicolumn{3}{|c|}{ Total population } & \multicolumn{3}{|c|}{ New cases } & \multicolumn{3}{|c|}{ Cancer deaths } \\
\hline & & Both sexes & Male & Female & Both sexes & Male & $\overline{\text { Female }}$ & Both sexes & Male & Female \\
\hline Beijing & 1 & $7,645,186$ & $3,859,586$ & $3,785,600$ & 23,339 & 11,784 & 11,555 & 13,544 & 7,969 & 5,575 \\
\hline Qianxi & 2 & 361,312 & 182,138 & 179,174 & 767 & 503 & 264 & 421 & 313 & 108 \\
\hline Shexian & 2 & 394,944 & 205,168 & 189,776 & 1,286 & 802 & 484 & 957 & 634 & 323 \\
\hline Cixian & 2 & 634,333 & 322,621 & 311,712 & 1,866 & 1,064 & 802 & 1,302 & 825 & 477 \\
\hline Baoding & 1 & 948,612 & 478,051 & 470,561 & 2,143 & 1,104 & 1,039 & 1,302 & 695 & 607 \\
\hline Yangquan & 1 & 683,165 & 346,023 & 337,142 & 1,403 & 807 & 596 & 913 & 582 & 331 \\
\hline Yangcheng & 2 & 383,165 & 192,119 & 191,046 & 1,272 & 728 & 544 & 785 & 504 & 281 \\
\hline Chifeng & 1 & $1,203,006$ & 613,725 & 589,281 & 2,051 & 1,186 & 865 & 1,325 & 797 & 528 \\
\hline Shenyang & 1 & $3,497,815$ & $1,722,976$ & $1,774,839$ & 10,801 & 5,598 & 5,203 & 6,891 & 4,051 & 2,840 \\
\hline Dalian & 1 & $2,266,224$ & $1,136,772$ & $1,129,452$ & 9,313 & 4,903 & 4,410 & 4,743 & 2,959 & 1,784 \\
\hline Zhuanghe & 2 & 915,660 & 461,826 & 453,834 & 2,314 & 1,310 & 1,004 & 1,539 & 972 & 567 \\
\hline An'shan & 1 & $1,471,775$ & 731,916 & 739,859 & 4,724 & 2,434 & 2,290 & 2,958 & 1,791 & 1,167 \\
\hline Benxi & 1 & 955,409 & 475,113 & 480,296 & 2,459 & 1,376 & 1,083 & 1,638 & 1,023 & 615 \\
\hline Dandong & 1 & 767,011 & 378,794 & 388,217 & 2,389 & 1,282 & 1,107 & 1,636 & 974 & 662 \\
\hline Donggang & 2 & 640,853 & 323,798 & 317,055 & 1,432 & 885 & 547 & 1,141 & 691 & 450 \\
\hline Dehui & 2 & 943,395 & 479,486 & 463,909 & 1,975 & 1,062 & 913 & 1,182 & 687 & 495 \\
\hline Yanji & 2 & 440,957 & 215,260 & 225,697 & 766 & 447 & 319 & 464 & 315 & 149 \\
\hline Daoli District, Harbin & 1 & 713,264 & 351,071 & 362,193 & 1,953 & 1,069 & 884 & 1,056 & 638 & 418 \\
\hline Nangang District, Harbin & 1 & $1,020,233$ & 508,921 & 511,312 & 2,389 & 1,246 & 1,143 & 1,660 & 1,005 & 655 \\
\hline Shangzhi & 2 & 616,046 & 314,864 & 301,182 & 1,254 & 724 & 530 & 653 & 410 & 243 \\
\hline Shanghai & 1 & $6,181,334$ & $3,084,496$ & $3,096,838$ & 25,366 & 13,321 & 12,045 & 16,933 & 9,840 & 7,093 \\
\hline Jintan & 2 & 545,000 & 262,407 & 282,593 & 1,561 & 987 & 574 & 1,242 & 838 & 404 \\
\hline Suzhou & 1 & $2,392,087$ & $1,183,716$ & $1,208,371$ & 8,381 & 4,838 & 3,543 & 4,504 & 2,835 & 1,669 \\
\hline Haian & 2 & 936,785 & 463,612 & 473,173 & 2,638 & 1,583 & 1,055 & 2,108 & 1,332 & 776 \\
\hline Qidong & 2 & $1,114,951$ & 548,805 & 566,146 & 3,516 & 2,172 & 1,344 & 2,928 & 1,899 & 1,029 \\
\hline Haimen & 2 & $1,016,228$ & 501,407 & 514,821 & 3,612 & 2,077 & 1,535 & 2,617 & 1,709 & 908 \\
\hline Lianyungang & 1 & 886,862 & 452,358 & 434,504 & 1,994 & 1,108 & 886 & 1,306 & 825 & 481 \\
\hline Donghai & 2 & $1,117,858$ & 579,751 & 538,107 & 2,083 & 1,283 & 800 & 1,506 & 979 & 527 \\
\hline Guanyun & 2 & $1,015,229$ & 534,502 & 480,727 & 1,995 & 1,204 & 791 & 1,596 & 1,068 & 528 \\
\hline Chuzhou District, Huai’an & 1 & $1,174,877$ & 609,088 & 565,789 & 2,828 & 1,728 & 1,100 & 1,925 & 1,179 & 746 \\
\hline Huaiyin District, Huai'an & 1 & 900,027 & 465,502 & 434,525 & 2,013 & 1,342 & 671 & 1,399 & 937 & 462 \\
\hline Xuyi & 2 & 759,450 & 388,180 & 371,270 & 1,764 & 1,097 & 667 & 1,077 & 678 & 399 \\
\hline Jinhu & 2 & 352,292 & 176,689 & 175,603 & 967 & 572 & 395 & 688 & 424 & 264 \\
\hline Sheyang & 2 & 965,817 & 494,682 & 471,135 & 3,052 & 1,734 & 1,318 & 2,213 & 1,388 & 825 \\
\hline Jianhu & 2 & 805,465 & 410,369 & 395,096 & 2,150 & 1,312 & 838 & 1,681 & 1,099 & 582 \\
\hline Dafeng & 2 & 724,147 & 363,326 & 360,821 & 2,014 & 1,167 & 847 & 1,597 & 975 & 622 \\
\hline Yangzhong & 2 & 272,046 & 134,758 & 137,288 & 1,043 & 576 & 467 & 873 & 532 & 341 \\
\hline Taixing & 2 & $1,128,840$ & 613,199 & 515,641 & 2,388 & 1,510 & 878 & 1,889 & 1,264 & 625 \\
\hline Hangzhou & 1 & $6,753,509$ & $3,403,893$ & $3,349,616$ & 22,625 & 12,690 & 9,935 & 11,592 & 7,571 & 4,021 \\
\hline Jiaxing & 1 & 509,367 & 253,819 & 255,548 & 1,564 & 853 & 711 & 912 & 573 & 339 \\
\hline Jiashan & 2 & 382,475 & 189,692 & 192,783 & 1,349 & 774 & 575 & 958 & 638 & 320 \\
\hline Haining & 2 & 653,957 & 322,969 & 330,988 & 1,666 & 915 & 751 & 994 & 638 & 356 \\
\hline Shangyu & 2 & 771,321 & 383462 & 387,859 & 2,127 & 1,345 & 782 & 1,466 & 981 & 485 \\
\hline Xianju & 2 & 490,070 & 255,438 & 234,632 & 1,282 & 813 & 469 & 998 & 675 & 323 \\
\hline Feixi & 2 & 858,895 & 449,882 & 409,013 & 1,955 & 1,346 & 609 & 1,269 & 920 & 349 \\
\hline Maanshan & 1 & 633,477 & 323,834 & 309,643 & 1,721 & 1,038 & 683 & 1,143 & 770 & 373 \\
\hline Tongling & 1 & 433,545 & 221,375 & 212,170 & 1,046 & 644 & 402 & 697 & 471 & 226 \\
\hline Changle & 2 & 673,717 & 355,091 & 318,626 & 1,474 & 872 & 602 & 828 & 569 & 259 \\
\hline Xiamen & 1 & 1160,135 & 583,873 & 576,262 & 3,851 & 2,255 & 1,596 & 2,145 & 1,448 & 697 \\
\hline
\end{tabular}


Table 1. Distribution of cancers for the total population, new cases, and deaths in each registry in 2009 (continued)

\begin{tabular}{|c|c|c|c|c|c|c|c|c|c|c|}
\hline \multirow{2}{*}{$\begin{array}{l}\text { Cancer } \\
\text { Registry }\end{array}$} & \multirow{2}{*}{$\begin{array}{c}\text { Category } \\
\text { 1: urban; 2: rural }\end{array}$} & \multicolumn{3}{|c|}{ Total population } & \multicolumn{3}{|c|}{ New cases } & \multicolumn{3}{|c|}{ Cancer deaths } \\
\hline & & Both sexes & Male & Female & Both sexes & Male & Female & Both sexes & Male & Female \\
\hline Zhanggong District, Ganzhou & 1 & 420,759 & 212,159 & 208,600 & 904 & 560 & 344 & 567 & 366 & 201 \\
\hline Linqu & 2 & 817,857 & 417,434 & 400,423 & 2,043 & 1,245 & 798 & 1,443 & 958 & 485 \\
\hline Wenshang & 2 & 762,828 & 388,454 & 374,374 & 1,405 & 873 & 532 & 1,130 & 724 & 406 \\
\hline Feicheng & 2 & 733,501 & 358,739 & 374,762 & 2,298 & 1,387 & 911 & 1,488 & 989 & 499 \\
\hline Yanshi & 2 & 602,266 & 306,192 & 296,074 & 1,117 & 583 & 534 & 748 & 429 & 319 \\
\hline Linzhou & 2 & $1,080,241$ & 557,392 & 522,849 & 2,744 & 1,462 & 1,282 & 1,701 & 1,057 & 644 \\
\hline Xiping & 2 & 858,002 & 434,899 & 423,103 & 1,628 & 926 & 702 & 1,258 & 767 & 491 \\
\hline Wuhan & 1 & $4,832,174$ & $2,484,622$ & $2,347,552$ & 12,590 & 6,978 & 5,612 & 6,961 & 4,504 & 2,457 \\
\hline Yunmeng & 2 & 524,801 & 261,237 & 263,564 & 942 & 558 & 384 & 767 & 503 & 264 \\
\hline Hengdong & 2 & 713,458 & 373,923 & 339,535 & 1,217 & 732 & 485 & 728 & 456 & 272 \\
\hline Guangzhou & 1 & $3,968,216$ & $2,014,580$ & $1,953,636$ & 13,062 & 7,169 & 5,893 & 8,133 & 5,093 & 3,040 \\
\hline Sihui & 2 & 413,363 & 211,351 & 202,012 & 947 & 563 & 384 & 601 & 400 & 201 \\
\hline Zhongshan & 1 & $1,468,391$ & 732,333 & 736,058 & 2,937 & 1,783 & 1,154 & 1,881 & 1,289 & 592 \\
\hline Liuzhou & 1 & $1,038,208$ & 533,050 & 505,158 & 2,435 & 1,396 & 1,039 & 1,357 & 862 & 495 \\
\hline Fusui & 2 & 444,332 & 236,000 & 208,332 & 759 & 525 & 234 & 529 & 391 & 138 \\
\hline Jiulongpo District, Chongqing & 1 & 798,618 & 402,961 & 395,657 & 1,458 & 914 & 544 & 1,220 & 841 & 379 \\
\hline Qingyang District, Chengdu & 1 & 534,701 & 277,154 & 257,547 & 1,434 & 845 & 589 & 880 & 583 & 297 \\
\hline Ziliujing District, Zigong & 1 & 357,600 & 179,873 & 177,727 & 916 & 597 & 319 & 462 & 330 & 132 \\
\hline Yanting & 2 & 610,103 & 316,499 & 293,604 & 2,317 & 1,481 & 836 & 1,850 & 1,177 & 673 \\
\hline Jingtai & 2 & 233,609 & 119,953 & 113,656 & 395 & 228 & 167 & 244 & 159 & 85 \\
\hline Liangzhou District, Wuwei & 1 & 990,583 & 524,276 & 466,307 & 2,837 & 1,886 & 951 & 2,024 & 1,382 & 642 \\
\hline Xining & 1 & 882,839 & 439,175 & 443,664 & 1,492 & 971 & 521 & 844 & 585 & 259 \\
\hline Xinyuan & 2 & 271,944 & 138,895 & 133,049 & 568 & 330 & 238 & 300 & 192 & 108 \\
\hline
\end{tabular}

Table 2. The incidences of cancers (ICD10:C00-C97) registered in China in 2009

\begin{tabular}{|c|c|c|c|c|c|c|}
\hline Areas & Gender & Cancer cases & Crude incidence $\left(1 / 10^{5}\right)$ & $\operatorname{ASRIC}\left(1 / 10^{5}\right)$ & ASRIW $\left(1 / 10^{5}\right)$ & Cumulative rate $(\%)$ \\
\hline \multirow[t]{3}{*}{ All areas } & Both sexes & 244,366 & 285.91 & 146.87 & 191.72 & 22.08 \\
\hline & Male & 137,462 & 317.97 & 165.92 & 220.33 & 25.68 \\
\hline & Female & 106,904 & 253.09 & 129.49 & 166.04 & 18.64 \\
\hline \multirow[t]{3}{*}{ Urban } & Both sexes & 174,418 & 303.39 & 150.31 & 195.74 & 22.23 \\
\hline & Male & 95,705 & 330.19 & 165.50 & 219.84 & 25.25 \\
\hline & Female & 78,713 & 276.15 & 137.09 & 175.03 & 19.44 \\
\hline \multirow[t]{3}{*}{ Rural } & Both sexes & 69,948 & 249.98 & 139.68 & 182.88 & 21.76 \\
\hline & Male & 41,757 & 293.10 & 166.94 & 220.94 & 26.65 \\
\hline & Female & 28,191 & 205.25 & 113.07 & 146.24 & 16.83 \\
\hline
\end{tabular}

ASRIC, age-standardized rate for the incidence based on the Chinese standard population; ASRIW, age-standardized rate for the incidence based on the world standard population; cumulative rate refers to the rate for all patients aged 0-74 years.

cancer registration areas, followed by gastric, colorectal, liver, and esophageal cancers. The 10 most common cancers accounted for $76.39 \%$ of all new cases, including $84.14 \%$ in males and $77.57 \%$ in females. Lung cancer was the most frequently diagnosed cancer in males, followed by gastric, liver, colorectal, and esophageal cancers. Breast cancer was the most frequently diagnosed cancer in females, followed by lung, colorectal, gastric, and liver cancers (Table 4). 
Table 3. Cancer mortalities in cancer registration areas in 2009

\begin{tabular}{|c|c|c|c|c|c|c|}
\hline Areas & Gender & Cancer deaths & Crude mortality $\left(1 / 10^{5}\right)$ & ASRMC $\left(1 / 10^{5}\right)$ & ASRMW $\left(1 / 10^{5}\right)$ & Cumulative rate $(\%)$ \\
\hline \multirow[t]{3}{*}{ All areas } & Both sexes & 154,310 & 180.54 & 85.06 & 115.65 & 12.94 \\
\hline & Male & 969,27 & 224.20 & 110.89 & 151.69 & 16.94 \\
\hline & Female & 573,83 & 135.85 & 60.53 & 82.18 & 9.06 \\
\hline \multirow[t]{3}{*}{ Urban } & Both sexes & 104,551 & 181.86 & 80.86 & 110.57 & 12.12 \\
\hline & Male & 647,68 & 223.45 & 104.57 & 143.96 & 15.71 \\
\hline & Female & 39,783 & 139.57 & 58.61 & 80.00 & 8.69 \\
\hline \multirow[t]{3}{*}{ Rural } & Both sexes & 49,759 & 177.83 & 94.40 & 126.73 & 14.78 \\
\hline & Male & 32,159 & 225.73 & 124.60 & 168.01 & 19.62 \\
\hline & Female & 17,600 & 128.14 & 64.93 & 87.08 & 9.89 \\
\hline
\end{tabular}

ASRMC, age-standardized rate for the mortality based on the Chinese standard population; ASRMW, age-standardized rate for the mortality based on the world standard population; cumulative rate refers to the rate for all patients aged 0-74 years.

Table 4. Incidences of the top 10 cancers in cancer registration areas in 2009

\begin{tabular}{|c|c|c|c|c|c|c|c|c|c|c|c|c|}
\hline \multirow{2}{*}{ Rank } & \multicolumn{4}{|c|}{ Both sexes } & \multicolumn{4}{|c|}{ Male } & \multicolumn{4}{|c|}{ Female } \\
\hline & Site & $\begin{array}{l}\text { Incidence } \\
\left(1 / 10^{5}\right)\end{array}$ & $\begin{array}{c}\text { Proportion }{ }^{\mathrm{a}} \\
(\%)\end{array}$ & $\begin{array}{l}\text { ASRIC } \\
\left(1 / 10^{5}\right)\end{array}$ & Site & $\begin{array}{l}\text { Incidence } \\
\left(1 / 10^{5}\right)\end{array}$ & $\begin{array}{c}\text { Proportion } \\
(\%)\end{array}$ & $\begin{array}{l}\text { ASRIC } \\
\left(1 / 10^{5}\right)\end{array}$ & Site & $\begin{array}{c}\text { Incidence } \\
\left(1 / 10^{5}\right)\end{array}$ & $\begin{array}{c}\text { Proportion }^{\mathrm{a}} \\
(\%)\end{array}$ & $\begin{array}{l}\text { ASRIC } \\
\left(1 / 10^{5}\right)\end{array}$ \\
\hline 1 & Lung & 53.57 & 18.74 & 25.34 & Lung & 70.40 & 22.14 & 34.75 & Breast & 42.55 & 16.81 & 23.16 \\
\hline 2 & Stomach & 36.21 & 12.67 & 17.85 & Stomach & 49.61 & 15.60 & 25.37 & Lung & 36.34 & 14.36 & 16.41 \\
\hline 3 & Colorectum & 29.44 & 10.30 & 14.21 & Liver & 41.99 & 13.21 & 22.49 & Colorectum & 26.42 & 10.44 & 12.29 \\
\hline 4 & Liver & 28.71 & 10.04 & 14.78 & Colorectum & 32.38 & 10.18 & 16.23 & Stomach & 22.50 & 8.89 & 10.62 \\
\hline 5 & Esophagus & 22.14 & 7.74 & 10.88 & Esophagus & 30.44 & 9.57 & 15.62 & Liver & 15.11 & 5.97 & 7.11 \\
\hline 6 & Breast & 21.21 & 7.42 & 11.64 & Prostate & 9.92 & 3.12 & 4.34 & Esophagus & 13.64 & 5.39 & 6.27 \\
\hline 7 & Pancreas & 7.28 & 2.55 & 3.35 & Bladder & 9.78 & 3.08 & 4.70 & Cervix & 12.96 & 5.12 & 7.42 \\
\hline 8 & Lymphoma & 6.68 & 2.34 & 3.75 & Pancreas & 8.24 & 2.59 & 4.01 & Thyroid gland & 10.09 & 3.99 & 6.50 \\
\hline 9 & Bladder & 6.61 & 2.31 & 3.03 & Lymphoma & 7.71 & 2.42 & 4.46 & Uterus & 8.77 & 3.46 & 4.69 \\
\hline 10 & Thyroid gland & 6.56 & 2.29 & 4.21 & Kidney & 7.07 & 2.22 & 3.82 & Ovary & 7.95 & 3.14 & 4.54 \\
\hline Top & & 218.40 & 76.39 & 109.05 & & 267.55 & 84.14 & 135.81 & & 196.32 & 77.57 & 99.01 \\
\hline
\end{tabular}

aProportion in all cancer incident cases. Other footnotes as in Table 2.

\section{Cancer mortalities of the 10 most common cancers}

Lung cancer was the leading cause of death in the cancer registration areas, followed by gastric, liver, esophageal, and colorectal cancers. The 10 most common cancers accounted for $84.27 \%$ of all cancer deaths. In males, lung cancer was the leading cause of death, followed by liver, gastric, esophageal, and colorectal cancers; in females, lung cancer was also the leading cause of death, followed by gastric, liver, colorectal, and breast cancers (Table 5).

\section{Discussion}

A recent goal of the National Health Care Reform Program of China is to establish more than 300 registries covering at least one tenth of the population of all rural areas. The year 2009 is the year that the Ministry of Health in China launched the National Program of Cancer Registry. Fifty-two new cancer registries were established based on 43 existing registries supported by central finance through the registry program. According to the NCCR's data submission requirements, 95 registries were expected to submit their 2009 cancer registration data in 2012. As of June 2012, 104 cancer registries had submitted data, a great increase compared with the previous year. In 2012, a total of 222 cancer registries recorded cancer data, covering 200 million people. The number of registries is expected to increase in the coming years. NCCR will focus on improving data quality and expanding the 
Table 5. Mortalities of the 10 most common cancers in cancer registration areas in 2009

\begin{tabular}{|c|c|c|c|c|c|c|c|c|c|c|c|c|}
\hline \multirow{2}{*}{ Rank } & \multicolumn{4}{|c|}{ Both sexes } & \multicolumn{4}{|c|}{ Male } & \multicolumn{4}{|c|}{ Female } \\
\hline & Site & $\begin{array}{l}\text { Mortality } \\
\left(1 / 10^{5}\right)\end{array}$ & $\begin{array}{c}\text { Proportion } \\
(\%)\end{array}$ & $\begin{array}{l}\text { ASRMC } \\
\left(1 / 10^{5}\right)\end{array}$ & Site & $\begin{array}{l}\text { Mortality } \\
\left(1 / 10^{5}\right)\end{array}$ & $\begin{array}{c}\text { Proportion }^{\mathrm{a}} \\
(\%)\end{array}$ & $\begin{array}{l}\text { ASRMC } \\
\left(1 / 10^{5}\right)\end{array}$ & Site & $\begin{array}{c}\text { Mortality } \\
\left(1 / 10^{5}\right)\end{array}$ & $\begin{array}{c}\text { Proportion }^{\mathrm{a}} \\
(\%)\end{array}$ & $\begin{array}{l}\text { ASRMC } \\
\left(1 / 10^{5}\right)\end{array}$ \\
\hline 1 & Lung & 45.57 & 25.24 & 20.61 & Lung & 61.00 & 27.21 & 29.15 & Lung & 29.77 & 21.91 & 12.58 \\
\hline 2 & Liver & 26.04 & 14.42 & 13.06 & Liver & 37.96 & 16.93 & 19.91 & Stomach & 16.91 & 12.45 & 7.19 \\
\hline 3 & Stomach & 25.88 & 14.33 & 11.86 & Stomach & 34.64 & 15.45 & 16.79 & Liver & 13.84 & 10.19 & 6.28 \\
\hline 4 & Esophagus & 16.77 & 9.29 & 7.75 & Esophagus & 23.29 & 10.39 & 11.42 & Colorectum & 12.69 & 9.34 & 5.09 \\
\hline 5 & Colorectum & 14.23 & 7.88 & 6.15 & Colorectum & 15.73 & 7.02 & 7.28 & Breast & 10.24 & 7.54 & 4.94 \\
\hline 6 & Pancreas & 6.61 & 3.66 & 2.98 & Pancreas & 7.45 & 3.32 & 3.59 & Esophagus & 10.11 & 7.44 & 4.22 \\
\hline 7 & Breast & 5.13 & 2.84 & 2.52 & lymphoma & 5.00 & 2.23 & 3.43 & Pancreas & 5.75 & 4.23 & 2.41 \\
\hline 8 & Leukemia & 4.28 & 2.37 & 2.88 & Leukemia & 4.59 & 2.05 & 2.37 & Gallbladder & 3.79 & 2.79 & 1.50 \\
\hline 9 & Brain & 3.87 & 2.15 & 2.29 & Prostate & 4.19 & 1.87 & 1.58 & Brain & 3.55 & 2.61 & 1.99 \\
\hline 10 & Lymphoma & 3.75 & 2.08 & 1.86 & Brain & 4.19 & 1.87 & 5.59 & Leukemia & 3.55 & 2.61 & 2.34 \\
\hline Top & 10 & 152.14 & 84.27 & 71.96 & & 198.04 & 88.33 & 98.11 & & 110.20 & 81.12 & 48.55 \\
\hline
\end{tabular}

coverage to improve the cancer surveillance system nationwide by establishing the foundations for cancer control.

To ensure the validity of accepted data, NCCR processes the data carefully according to the national criteria issued in the program protocol. The incidence, mortality, and population data must be reasonable compared with the levels for similar populations, for example, those with a similar location, socioeconomic status, and lifestyle. The indicators of completeness and validity, such as $\mathrm{MV} \%$, DCO\%, $\mathrm{M} / \mathrm{I}$ ratio, $\mathrm{UB} \%$, and O\&U\%, were evaluated for every registry's data. Through double evaluations at the provincial and national levels, 72 registries qualified for inclusion, and 32 were considered invalid. The valid data were pooled and analyzed to create the final results for the 2009 annual report.

The cancer incidence and mortality statistics for 2009 were very close to those for $2008^{[6]}$. Although the included registries were quite different from those that were included in the 2008 report, the overall cancer incidence and mortality data for the two years were reasonably stable, indicating that the pooled data were valid and could represent the cancer burden at the national level. The representativeness of the cancer registry for different groups, such as urban and rural populations and various regions, should be evaluated ${ }^{[7]}$.

The cancer patterns differ considerably between urban and rural areas in China ${ }^{[8]}$. In urban areas, lung cancer, female breast cancer, and colorectal cancer are major cancers with higher incidences than in rural areas. However, cancers of the digestive system, such as esophageal cancer, gastric cancer, and liver cancer, are common in rural areas. The overall cancer incidence in urban areas is higher and mortality is lower than those in rural areas ${ }^{[8,9]}$. This difference is the result of limited medical resources, a relatively low level of cancer diagnosis and treatment, and a lack of health education in rural areas. In urban areas, the cancer spectrum is tending toward the characteristics of developed countries. The burdens of lung cancer, colorectal cancer, and female breast cancer continue to increase. Cancer in rural areas still retains the cancer patterns of developing countries. Thus, the emphasis of cancer control should consider these differences and implement efficient strategies based on cancer surveillance results.

Currently, the Ministry of Health is developing an action plan for preventing and controlling noncommunicable diseases in the twelfth 5-year plan. Cancer is a major disease seriously threatening people's health in China. The emphasis in rural areas would focus on professional training in primary care centers, health education/promotion, and early diagnosis/treatment, especially for cancers that are common in rural areas, such as esophageal, gastric, cervical, and liver cancers. In cities, behavioral interventions, such as tobacco control and healthy lifestyle promotion, should be enhanced, and high-risk groups should undergo cancer screening to achieve the goal of reducing cancer mortality within a short time.

Received: 2013-01-18; revised: 2013-01-30; accepted: 2013-01-30. 


\section{References}

[1] Parkin DM. The evolution of the population-based cancer registry. Nat Rev Cancer, 2006,6:603-612.

[2] National Office for Cancer Prevention and Control, National Center for Cancer Registry, Disease Prevention and Control Bureau, MOH. Chinese Cancer Registry Annual Report (2010). Beijing: Military Medical Science Press, 2011.

[3] Larsen IK, Småstuen M, Johannesen TB, et al. Data quality at the Cancer Registry of Norway: an overview of comparability, completeness, validity and timeliness. Eur J Cancer, 2009,45: 1218-1231.

[4] Curado MP, Edwards B, Shin HR, et al. eds. Cancer Incidence in Five Continents. Vol. IX. IARC Scientific Publications No.160. Lyon: IARC, 2008.

[5] Ferlay J. The IARC crgTools Programs. Lyon: IACR; 2006. Available online: http://www.iacr.com.fr/iarccrgtools.htm.

[6] National Office for Cancer Prevention and Control, National Center for Cancer Registry, Disease Prevnetion and Control Bureau, MOH. Chinese Cancer Registry Annual Report (2011). Beijing: Military Medical Science Press, 2011.

[7] Li GL, Chen WQ. Representativeness of population-based cancer registration in China-comparison of urban and rural areas. Asian Pac J Cancer Prev, 2009, 10:559-564.

[8] Chen WQ, Zheng RS, Zeng HM, et al. Trend analysis and projection of cancer incidence in China between 1989 and 2008. Zhonghua Zhong Liu Za Zhi, 2012,34:517 -524. [in Chinese]

[9] Zeng HM, Zheng RS, Zhang SW, et al. Trend analysis of cancer mortality in China between 1989 and 2008. Zhonghua Zhong Liu Za Zhi, 2012,34:525-531. [in Chinese] 